Research Article

\title{
An Extension Failure Criterion for Brittle Rock
}

\author{
Aizhong Lu, ${ }^{1}$ Ning Zhang $\mathbb{D}^{1}{ }^{1}$ and Guisen Zeng ${ }^{2}$ \\ ${ }^{1}$ Institute of Hydroelectric and Geotechnical Engineering, North China Electric Power University, Beijing 102206, China \\ ${ }^{2}$ Electric Power Research Institute of Guizhou Power Grid Co., Ltd., Guiyang, Zunyi, Guizhou 550002, China
}

Correspondence should be addressed to Ning Zhang; zning1125@ncepu.edu.cn

Received 4 August 2020; Revised 4 October 2020; Accepted 11 October 2020; Published 28 October 2020

Academic Editor: Fengqiang Gong

Copyright (c) 2020 Aizhong Lu et al. This is an open access article distributed under the Creative Commons Attribution License, which permits unrestricted use, distribution, and reproduction in any medium, provided the original work is properly cited.

Under the triaxial compressive state, the compressive strain is supposed to happen in the direction of the maximum principal stress, but tensile strain happens in the direction of the minimum principal stress. Moreover, as the intermediate principal stress is not too high, the corresponding strain can also be tensile. If the brittle rock is assumed as linear elastic in the prefailure stage, a new strength criterion based on the sum of the two tensile strains was presented. The new criterion considers the differences in mechanical parameters (i.e., elastic modulus and Poisson's ratio) under tension and compression. The parameters of the criterion only include Poisson's ratio and uniaxial strength. And the effect of the intermediate principal stress $\sigma_{2}$ can be reflected. Certain featured failure phenomenon of rock material can be explained well by the proposed criterion. The results of conventional and true triaxial tests can verify the criterion well. Finally, the criterion is compared with the Mohr-Coulomb and Drucker-Prager criteria.

\section{Introduction}

Most of the common-used failure criteria for rock material describe shear failure. Coulomb developed the first linear failure criterion that reflects the shear failure feature [1]. The nonlinear shear failure criterion on the basis of the envelope of all of the stress circles that correspond to the failure stress state was proposed by Mohr [2]. The Mohr-Coulomb criterion is the most commonly used in practice. Some empirical failure criteria that follow Mohr's hypothesis can be expressed in the functional form $\tau=f_{1}(\sigma)$ or $\sigma_{1}=f_{2}\left(\sigma_{3}\right)$ [3]. The Hoek-Brown criterion is one such failure law $[4,5]$. Based on the Mohr-Coulomb criterion and Hoek-Brown criterion, some criteria considering more influence factors were presented. For example, Singh and Rao [6] presented the modified Mohr-Coulomb criterion for nonlinear triaxial and polyaxial strength of intact rocks. Gong et al. $[5,7]$ established a dynamic strength criterion and verified the scope of application through experiments. Ma et al. [8] proposed a new three-dimensional failure criterion combining the Mogi criterion and the generalized Hoek-Brown criterion.

Mohr's theory of failure believes that failure is just related to the maximum and minimum principal stresses, $\sigma_{1}$ and $\sigma_{3}$, and has nothing to do with the intermediate principal stress $\sigma_{2}$, whereas most true-triaxial tests $\left(\sigma_{2} \neq \sigma_{3}\right)$ show that the intermediate principal stress $\sigma_{2}$ does have a significant influence on the value of $\sigma_{1}$ at failure [9]. For the true triaxial stress states (i.e., $\sigma_{1}>\sigma_{2}>\sigma_{3}$ ), several failure criteria have been developed to consider the influence of the intermediate principal stress $\sigma_{2}$ [9-13].

Under triaxial compression, the failure of brittle rock includes the process of microcrack initiating, extending, and coalescing. And the macrotension failure can usually be observed during lateral extending deformation, especially as the value of $\sigma_{2}+\sigma_{3}$ is relatively small (Figure 1) [3, 14], whereas this kind of extending tension failure can hardly be reflected by the criteria used commonly.

Figure 1 illustrates the complete stress-strain curve for a rock under compression, which can be divided conceptually into four regions. In region $\mathrm{AB}$, some visible microcracks appear with the axes parallel (within $\pm 10^{\circ}$ ) to the direction of $\sigma_{1}$. In region $\mathrm{BC}$, the number of microcracks increases rapidly. At the point of the ultimate specimen strength, a macroscopic fracture plane develops in the central portion of the specimen. With further compression, this fracture plane grows towards one or both ends of the specimen by stepwise joining at existing microcracks. At the end of region $\mathrm{BC}$, the 


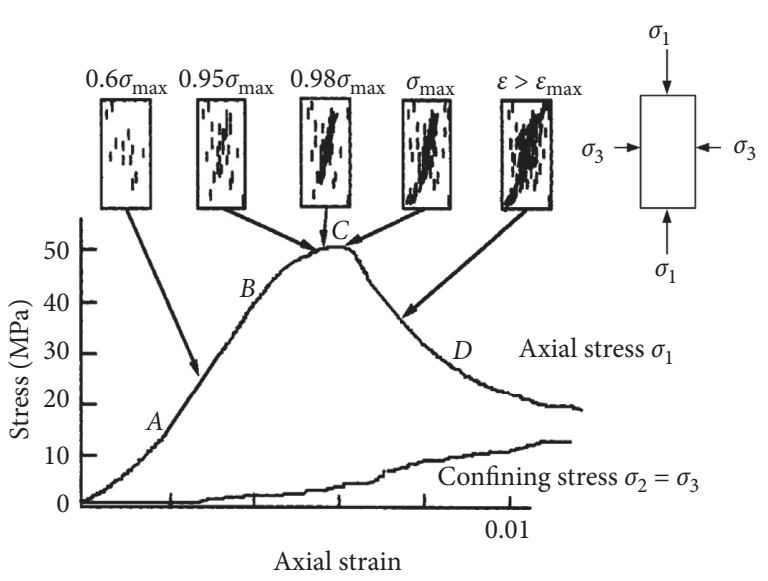

FIGURE 1: The stress-strain curve of argillaceous quartzite specimens and cartoons of the state of microcracking observed on specimens [14].

microcracks begin to coalesce along a plane and form a macroscopic fracture "plane" at the failure point C. Finally, in region $\mathrm{CD}$, the fracture plane extends through the entire specimen [3].

It can be observed from Figure 1 that, before the macroscopic fracture "plane," lateral extension failure has occurred due to plenty of longitudinal microfractures. The criterion presented herein is developed for this situation. As mentioned above, lateral tension strain is observed at failure. So, can we use the maximum tensile strain to develop the failure criterion? The answer is Yes for conventional triaxial condition, $\sigma_{2}=\sigma_{3}$, but No for the true triaxial condition, $\sigma_{1}>\sigma_{2}>\sigma_{3}$. For the conventional triaxial test, Fujii et al. found that the critical lateral tensile strain keeps as a constant, relatively unaffected by the confining pressure at failure [15]. This kind of rock failure follows the maximum tensile strain criterion. But for the true triaxial test, under a certain value of $\sigma_{3}$, the maximum tensile strain $\varepsilon_{1}$ in the $\sigma_{3}$ direction increases with increasing $\sigma_{2}$ (Figure 2). If the maximum tensile strain criterion is available here, that is, the value of $\varepsilon_{1}$ should be a constant, the value of $\sigma_{1}$ at failure should decrease with increasing $\sigma_{2}$, which is not in line with most test data. Thus, the maximum tensile strain criterion cannot be used for the condition of true triaxial compression. Under this background, this study tried to present a new extension failure criterion for brittle rock. This extension criterion can reflect the effect of the intermediate principal stress. And certain featured failure phenomenon of rock material, like yielding which will happen under the hydrostatic tension but not under the hydrostatic compression, can also be explained well by the proposed criterion.

Under the triaxial compressive stress state, as the value of $\sigma_{1}$ is relatively small, the strains in the $\sigma_{2}$ and $\sigma_{3}$ directions are both tensile, which differs from the tensile strain under uniaxial tension. It is not suitable to use the maximum tensile strain criterion in this case. It is believed herein that the rock failure depends not only on the maximum tensile strain $\varepsilon_{1}$ but also on the intermediate principal tensile strain $\varepsilon_{2}$. Then a new strength criterion is established by the value
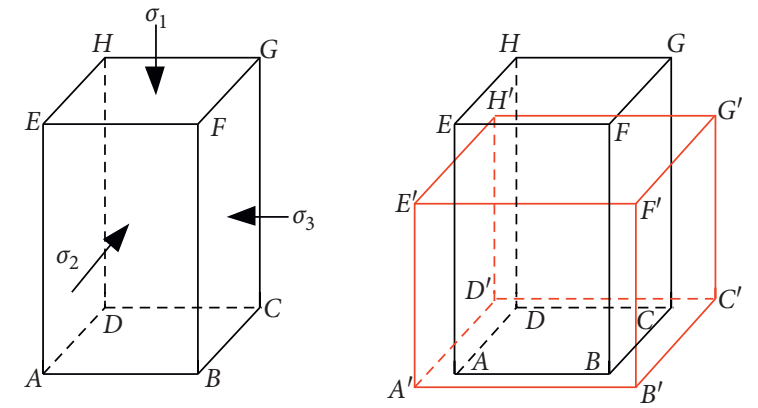

Figure 2: Cubic specimen under true triaxial stress.

of $\varepsilon_{1}+\varepsilon_{2}$. The new criterion is actually the same as the maximum tensile strain criterion just for the conventional test (i.e., $\sigma_{2}=\sigma_{3}$ ) (see Section 5.1). In the following section, we know that, under the small deformation theory, $\varepsilon_{1}+\varepsilon_{2}$ actually denotes the extension rate of the $\sigma_{1}$-plane, that is, the lateral deformation. For brittle rock, the theoretical results by the new criterion agree with the test results well.

\section{Theoretical Basis}

2.1. Tensile Strain under Triaxial Compression. In underground rock engineering, the three principal stresses in most areas are compression. Thus, the case of all three principal compressive stresses is discussed here. Compressive stress is considered positive, and compressive strain is taken as positive. Under this sign convention, one has $\sigma_{1} \geq \sigma_{2} \geq$ $\sigma_{3} \geq 0$, and the corresponding strains are the maximum, intermediate, and minimum principal strains, $\varepsilon_{1}, \varepsilon_{2}$, and $\varepsilon_{3}$ (i.e., $\varepsilon_{1} \geq \varepsilon_{2} \geq \varepsilon_{3}$ ). According to Figure 2, the three principal strains can be given by

$$
\begin{aligned}
& \varepsilon_{1}=\frac{A^{\prime} E^{\prime}-\mathrm{AE}}{\mathrm{AE}}, \\
& \varepsilon_{2}=\frac{B^{\prime} C^{\prime}-\mathrm{BC}}{\mathrm{BC}}, \\
& \varepsilon_{3}=\frac{A^{\prime} B^{\prime}-\mathrm{AB}}{\mathrm{AB}} .
\end{aligned}
$$

Brittle rock material can be assumed as isotropic and linear elastic in prefailure stage [16]. For the isotropic rock material, the maximum principal strain $\varepsilon_{1}$ must be in the $\sigma_{1}$ direction, the intermediate principal strain $\varepsilon_{2}$ in the $\sigma_{2}$ direction, and the minimum principal strain $\varepsilon_{3}$ in the $\sigma_{3}$ direction. Based on the sign convention and Hooke's law, one has

$$
\begin{aligned}
& \varepsilon_{1}=\frac{1}{E_{c}}\left[\sigma_{1}-\mu_{c}\left(\sigma_{2}+\sigma_{3}\right)\right], \\
& \varepsilon_{2}=\frac{1}{E_{c}}\left[\sigma_{2}-\mu_{c}\left(\sigma_{1}+\sigma_{3}\right)\right], \\
& \varepsilon_{3}=\frac{1}{E_{c}}\left[\sigma_{3}-\mu_{c}\left(\sigma_{2}+\sigma_{1}\right)\right],
\end{aligned}
$$


where $E_{c}$ and $\mu_{c}$ are Young's modulus and Poisson's ratio under uniaxial compressive stress, respectively. The values of the two parameters can be tested by a uniaxial compressive test.

It can be proved from equations (4)-(6) that, as $\sigma_{1}>\sigma_{3} / \mu_{c}-\sigma_{2}, \quad \varepsilon_{3}<0$, and as $\sigma_{1} \geq \sigma_{2} / \mu_{c}-\sigma_{3}, \quad \varepsilon_{3}<0$ and $\varepsilon_{2} \leq 0$. This shows that, even under the triaxial compressive stress state, the tensile strain will still happen due to the Poisson effect on the directions of the minimum and/or intermediate principal stresses. One can say that tensile strain is not always caused by tensile stress.

2.2. Elastic Parameters of Uniaxial Compression. The case of uniaxial compression means that the two smaller principal stresses $\sigma_{2}$ and $\sigma_{3}$ equal zero, with just the nonzero maximum principal stress $\sigma_{1}$. In this case, we usually use $\sigma_{c}$ denoting the uniaxial compressive stress. Then, one has

$$
\begin{aligned}
& E_{c}=\frac{\sigma^{c}}{\left|\varepsilon_{1}\right|}, \\
& \mu_{c}=\left|\frac{\varepsilon_{3}}{\varepsilon_{1}}\right|=\left|\frac{\varepsilon_{2}}{\varepsilon_{1}}\right| .
\end{aligned}
$$

For the isotropic linear material, $\varepsilon_{2}=\varepsilon_{3}$ and $0<\mu_{c}<0.5$. The values of $\varepsilon_{1}, \varepsilon_{2}$, and $\varepsilon_{3}$ in equations (7) and (8) can also be calculated by equations (1)-(3), only with the lengths of $A^{\prime} B^{\prime}, A B$, and so on, being determined by the uniaxial compression test. The strain $\varepsilon_{1}$ in the $\sigma$ direction must be compressive (i.e., $\varepsilon_{1}>0$ ), and the lateral strains in the $\sigma$-plane must be tensile $\left(\varepsilon_{2}=\varepsilon_{3}<0\right)$.

2.3. Elastic Parameters of Uniaxial Tension. For the uniaxial tension test, $\sigma^{t}$ is the uniaxial tensile stress (see Figure 3 ). The values of $\varepsilon_{1}, \varepsilon_{2}$, and $\varepsilon_{3}$ can, respectively, be given by

$$
\begin{aligned}
& \varepsilon_{1}=\frac{B C-B^{\prime} C^{\prime}}{B C}>0, \\
& \varepsilon_{2}=\frac{A B-A^{\prime} B^{\prime}}{A B}>0, \\
& \varepsilon_{3}=\frac{A E-A^{\prime} E^{\prime}}{A E}<0 .
\end{aligned}
$$

Let $E_{t}$ and $\mu_{t}$ denote Young's modulus and Poisson's ratio under uniaxial tension, respectively. From Figure 3, one has

$$
\begin{aligned}
& E_{t}=\frac{\sigma^{t}}{\left|\varepsilon_{3}\right|}, \\
& \mu_{t}=\left|\frac{\varepsilon_{1}}{\varepsilon_{3}}\right|=\left|\frac{\varepsilon_{2}}{\varepsilon_{3}}\right| .
\end{aligned}
$$

For the isotropic linear material, $\varepsilon_{2}=\varepsilon_{3}$ and $0<\mu_{t}<0.5$. The values of $\varepsilon_{1}, \varepsilon_{2}$, and $\varepsilon_{3}$ in equations (12) and (13) can also be calculated by equations (9)-(11). For this condition, the strain $\varepsilon_{3}$ in the $\sigma$ direction must be tensile (i.e., $\varepsilon_{3}<0$ ), and
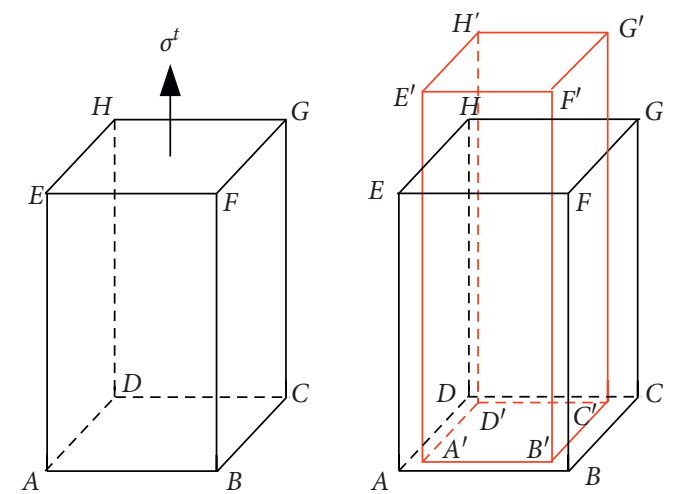

Figure 3: Cubic specimen under uniaxial tensile stress.

the lateral strains in the $\sigma$-plane must be compressive $\left(\varepsilon_{2}=\varepsilon_{3}>0\right)$.

\section{A New Strength Criterion}

3.1. Physical Meaning of $\varepsilon_{1}+\varepsilon_{2}$. From Section 2.1, under triaxial compression state, the strains in the $\sigma_{2}$ and $\sigma_{3}$ directions should be $\varepsilon_{2}$ and $\varepsilon_{3}$, respectively. Moreover, $\varepsilon_{2}$ and $\varepsilon_{3}$ are both tensile strains as $\sigma_{1}$ is relatively great. For small deformation theory, the value of $\varepsilon_{2}+\varepsilon_{3}$ actually denotes the extension rate of the $\sigma_{1}$-plane (see Figure 4 ).

As shown in Figure 4, $\mathrm{S}$ is the initial area of the crosssection $\mathrm{ABCD}$ before the load is applied. And $\Delta S$ denotes the area increase after the load is applied. Then, the extension rate of the $\sigma_{1}$-plane can be calculated by

$$
\begin{aligned}
\frac{\Delta S}{S} & =\frac{\left(l+\Delta l_{1}\right)\left(l+\Delta l_{2}\right)-l^{2}}{l^{2}} \\
& =\frac{\Delta l_{1}}{l}+\frac{\Delta l_{2}}{l}+\frac{\Delta l_{1}}{l} \frac{\Delta l_{2}}{l} \\
& =\varepsilon_{2}+\varepsilon_{3}+\varepsilon_{2} \varepsilon_{3} \\
& =\varepsilon_{2}+\varepsilon_{3} .
\end{aligned}
$$

3.2. Strength Criterion. The uniaxial tensile strength (UTS) of rock $\sigma_{t}$ is the stress at failure under the uniaxial tensile test. The critical tensile strain corresponding to $\sigma_{t}$ is $\varepsilon_{u}$. For linear-elastic material in prefailure stage, one has

$$
\varepsilon_{u}=\frac{\sigma_{t}}{E_{t}} .
$$

Based on the maximum strain theory, the failure will occur if one of the strains in the natural axes exceeds the corresponding allowable strain $\varepsilon \mathrm{u}$. In this work, we consider both strains occurring in the principal stress plane, that is, $\varepsilon_{2}+\varepsilon_{3}$. As proved in Section 3.1, the meaning of the sum of $\varepsilon_{2}$ and $\varepsilon_{3}$ is the extension rate of the responding plane. In this way, it is supposed that failure will occur as $\varepsilon_{2}+\varepsilon_{3}$ reaches twice the maximum uniaxial tensile strain; that is, 

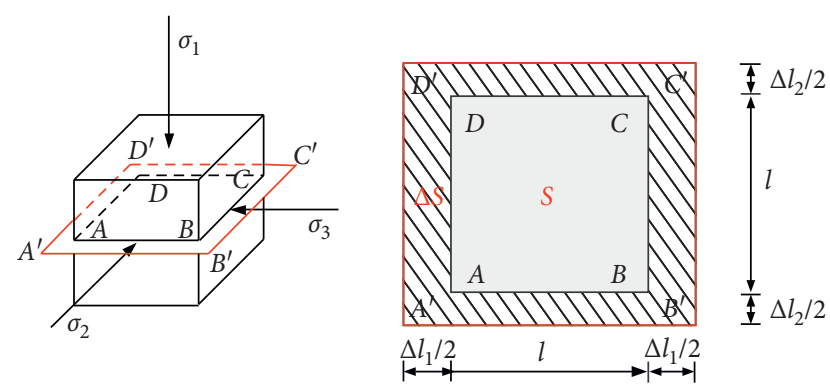

FIgURE 4: Cross-section deformation.

$$
\begin{aligned}
\varepsilon_{2}+\varepsilon_{3} & =2 \varepsilon_{u} \\
& =2 \frac{\sigma_{t}}{E_{t}} .
\end{aligned}
$$

Substituting equations (5) and (6) into equation (16) gives

$$
\sigma_{1}=\frac{1-\mu_{c}}{2 \mu_{c}}\left(\sigma_{2}+\sigma_{3}\right)-\frac{E_{c} \sigma_{t}}{E_{t} \mu_{c}} .
$$

Equation (17) is the general expression of the failure criterion.

Many experimental data show that elastic parameters of rock material are different in compression and tensile condition [17-24]. The ratio of the compressive elastic modulus $E_{c}$ to the tensile elastic modulus $E_{t}$ is mainly within $1-10$ or even up to 18 for a special case. Poisson's ratio $\mu_{c}$ under the compression condition is bigger than $\mu_{t}$ under the tension condition. The value of $\mu_{t}$ is between 0.05 and 0.3 , mostly around 0.1 for rock materials.

For the uniaxial compressive state, $\sigma_{1}=\sigma_{c}$ and $\sigma_{2}=\sigma_{3}=0$, where $\sigma_{c}$ is the uniaxial compressive strength (UCS). The criterion equation (17) can be simplified as

$$
\sigma_{t}=-\frac{\mu_{c} E_{t}}{E_{c}} \sigma_{c} .
$$

Equation (18) gives the relationship between the two strengths $\sigma_{c}$ and $\sigma_{t}$.

For most materials, the mechanical parameters mainly obey the following relationship [25]:

$$
\frac{\mu_{c}}{E_{c}}=\frac{\mu_{t}}{E_{t}} .
$$

Thus, equation (18) becomes

$$
\sigma_{t}=-\mu_{t} \sigma_{c}
$$

Substituting equations (19) and (20) into equation (17) gives

$$
\sigma_{1}=\sigma_{c}+\frac{1-\mu_{c}}{2 \mu_{c}}\left(\sigma_{2}+\sigma_{3}\right) .
$$

The proposed failure criterion is equation (21), in which two mechanical parameters (i.e., UCS $\sigma_{c}$ and Poisson's ratio $\left.\mu_{c}\right)$ are contained. If we ignore equation (19), the criteria will need one more parameter.
From Section 3.1, the criterion indicates that tensile failure in rock will happen as the extension rate of the $\sigma_{1}$-plane reaches a critical value. Moreover, the mechanism of tensile failure can be explained as the $\sigma_{1}$-plane extension is the result of the microcracks distributed in mineral grains, and as mineral grains get separated from each other, macroscopic failure happens.

\section{Analysis and Discussion}

4.1. Application Condition. The criterion equation (21) is obtained based on equation (16). Equation (16) shows that $\varepsilon_{2}+\varepsilon_{3}$ should be smaller than zero. Combining equations (5) and (6), one has

$$
\sigma_{2}+\sigma_{3}<\frac{2 \mu_{c}}{1-\mu_{c}} \sigma_{1} .
$$

Equation (22) is the application condition of the criterion equation (21). The physical meaning of the application condition can be explained as follows. As the lateral pressure is relatively small (i.e., $\sigma_{2}+\sigma_{3}<2 \mu_{c} \sigma_{1} /\left(1-\mu_{c}\right)$ ), the cross section in the $\sigma_{1}$-plane will extend outwards, and as the extension reaches a critical value, tensile failure will happen, while as the lateral pressure is relatively great (i.e., $\left.\sigma_{2}+\sigma_{3}<2 \mu_{c} \sigma_{1} /\left(1-\mu_{c}\right)\right)$, the lateral cross section will shrink inwards and the volume of the cubic will contract consequently; in this condition, tensile failure will not happen. It may be the other failure modes like a shear failure, which cannot be described by this criterion anymore.

4.2. Uniaxial Tensile Strength (UTS). The tensile strength is an important mechanical parameter in rock mechanics. However, it is difficult to conduct the uniaxial tensile test on rock materials, and the obtained data always show significant discreteness [26]. The experimental data and conclusions in the literature are so contrasting that some authors have even suggested that the tensile strength should not be considered as a material property [27]. Numerous indirect tests have been devised as an alternative to the direct pull test to study the tensile strength.

It can be seen from equation (20) that the uniaxial tensile strength of rock can be estimated by $\mu_{t}$ and $\sigma \mathrm{c}$. As mentioned earlier, for rock material, the values of $\mu_{t}$ are between 0.05 and 0.3 , mostly around 0.1 . So the uniaxial compressive strength is 3 to 20 times of the uniaxial tensile strength, mostly about 10 times, which is consistent with the actual appearance [28]. It is easier to test tensile Poisson's ratio and uniaxial compressive strength; thus, the uniaxial tensile strength can be obtained by equation (20). Furthermore, for the given elastic modulus (i.e., $E_{c}$ and $E_{t}$ ) and tensile Poisson's ratio, the more accurate uniaxial tensile strength can be obtained by equation (18).

4.3. Hydrostatic Stress. Will rock failure occur under the hydrostatic compression? We assumed the answer is Yes and the value of yield compressive stress is $\sigma_{c}^{0}$ (i.e., $\left.\sigma_{1}=\sigma_{2}=\sigma_{3}=\sigma_{c}^{0}\left(\sigma_{c}^{0}>0\right)\right)$. From equation (21), we have 


$$
\sigma_{c}^{0}=\frac{\mu_{c}}{2 \mu_{c}-1} \sigma_{c}
$$

Considering that $2 \mu_{c}-1<0$, so $\sigma_{c}^{0}<0$, which is in contradiction with the assumption. It indicates that rock material will not fail under the hydrostatic compressive stress, because no tensile strain occurs under hydrostatic compression. This conclusion agrees with the test results.

As for the hydrostatic tension state, the value of $\varepsilon_{2}+\varepsilon_{3}$ will increase with increasing hydrostatic tension $\sigma_{t}^{0}$. From Hooke's law and equation (16), it can be proved that failure will occur in rock material as $\sigma_{t}^{0}=\sigma_{t} /\left(1-2 \mu_{t}\right)$, meaning that hydrostatic tension can cause a failure which has been verified by the test as well.

4.4. Yield Curve in Deviatoric Plane. In this section, the yield curve of equation (21) on the $\pi^{\prime}$-plane is to be discussed. The $\pi^{\prime}$-plane can be expressed by

$$
\sigma_{1}+\sigma_{2}+\sigma_{3}=\sigma_{c}
$$

The $\pi^{\prime}$-plane is parallel to the $\pi$-plane (i.e., $\sigma_{1}+$ $\sigma_{2}+\sigma_{3}=0$ ), with the same intercepts $\sigma_{c}$ in three axes. Combining equations (21) and (24), equations

$$
\left\{\begin{array}{l}
x=\frac{1}{\sqrt{2}}\left(\sigma_{1}-\sigma_{3}\right) \\
y=\frac{1}{\sqrt{6}}\left(2 \sigma_{2}-\sigma_{1}-\sigma_{3}\right)
\end{array}\right.
$$

and the symmetry of the yield curves, the three yield curves/ lines on the $\pi^{\prime}$-plane, respectively, are

$$
\begin{aligned}
& y=\sqrt{3} x-2 \sqrt{\frac{2}{3}} \sigma_{c}, \\
& y=-\sqrt{3} x-2 \sqrt{\frac{2}{3}} \sigma_{c}, \\
& y=\sqrt{\frac{2}{3}} \sigma_{c} .
\end{aligned}
$$

The yield curve by equations (26)-(28) is a regular triangle with the side of $\sqrt{2} \sigma_{c}$ (see Figure 5). The shape and size of the yield curves are only related to the UCS $\sigma_{c}$, having nothing to do with $\mu_{c}$. Moreover, the shape of equation (21) in principal stress space is a triangular pyramid.

4.5. Effect of Intermediate Principal Stress. For a long time, there has been no clear understanding of the effect of the intermediate principal stress on rock strength. Some suggested that the effect can be ignored. While some others had the opposite view, they believe that the intermediate principal stress has a great impact on strength of rock material. To solve this problem, many true triaxial tests of rocks have been conducted.

From equations (21) and (22), for the case of $\sigma_{2}+\sigma_{3}<2 \mu_{c} \sigma_{1} /\left(1-\mu_{c}\right)$, one has $\sigma_{1}=\sigma_{c}+\left(1-\mu_{c} / 2 \mu_{c}\right)$ $\left(\sigma_{2}+\sigma_{3}\right)$. Obviously, the stress $\sigma_{1}$ at failure (i.e., the strength) is proportional to the intermediate principal stress $\sigma_{2}$, with the influence coefficient being $1-\mu_{c} / 2 \mu_{c}$, which is in line with the experimental data as the value of is $\sigma_{2}$ not too great, whereas the strength $\sigma_{1}$ decreases instead under greater $\sigma_{2}$, which cannot be explained using the criterion. The reason behind this may be that the failure mode under greater $\sigma_{2}$ is not tensile. Thus, the proposed criterion is not applicable anymore.

4.6. Comparison with Other Criteria. The Mohr-Coulomb (M-C) and Drucker-Prager (D-P) criteria are commonly used in geotechnical engineering. Thus, we compare the proposed criterion (hereafter, referred to as LU criterion) with the two classical criteria.

$\mathrm{M}-\mathrm{C}$ criterion can be given as

$$
\sigma_{1}=\frac{1+\sin \varphi}{1-\sin \varphi} \sigma_{3}+\frac{2 c \cos \varphi}{1-\sin \varphi}=\frac{1+\sin \varphi}{1-\sin \varphi} \sigma_{3}+\sigma_{c},
$$

where $c, \varphi$ are the cohesion and angle of internal friction of rock, respectively.

On the $\pi^{\prime}$-plane, the M-C criterion has six yield curves/ lines. And the three on the right-hand side of the $y$-axis are

$$
\begin{aligned}
& y=\frac{1-\sin \varphi}{3+\sin \varphi} \sqrt{3} x-\frac{3-\sin \varphi}{3+\sin \varphi} \sqrt{\frac{2}{3}} \sigma_{c}, \\
& y=-\frac{1}{\sin \varphi} \sqrt{3} x+\frac{3-\sin \varphi}{2} \sqrt{\frac{2}{3}} \sigma_{c}, \\
& y=-\frac{1+\sin \varphi}{3-\sin \varphi} \sqrt{3} x+\sqrt{\frac{2}{3}} \sigma_{c}
\end{aligned}
$$

which are symmetric with the other three on the left-hand side.

The yield curves of M-C and D-P criteria on the $\pi^{\prime}$-plane are hexagon and circle, respectively. As the three curves of uniaxial compression tests coincide with each other on the $\pi^{\prime}$-plane, the three yield surfaces in principal stress space are plotted in Figure 6. The LU curve encircles the Drucker-Prager curve, while the Drucker-Prager curve encircles the Mohr-Coulomb curve, reflecting that the Mohr-Coulomb criterion is the most conservative one, the Drucker-Prager criterion is less, and the LU criterion is the least one among them.

\section{Experimental Verification}

5.1. Conventional Triaxial Test. The failure behavior of rock materials has been intensely studied under triaxial compression with two of the principal stresses being equal (i.e., $\sigma_{2}=\sigma_{3}$, confining pressure) on cylindrical specimens, which is known as the conventional triaxial test.

From equations (4) and (5), if $\sigma_{2}=\sigma_{3}$, one has $\varepsilon_{2}=\varepsilon_{3}$, and as $\sigma_{1}>\left(\sigma_{2} / \mu_{c}\right)-\sigma_{3}, \varepsilon_{2}$ and $\varepsilon_{3}$ are both the maximum tensile strains. For a cylinder specimen, $\varepsilon_{2}$ and $\varepsilon_{32}$ are the radial and tangential strains, respectively. Thus, for the conventional triaxial test, the radial strain is the same as the tangential strain. 

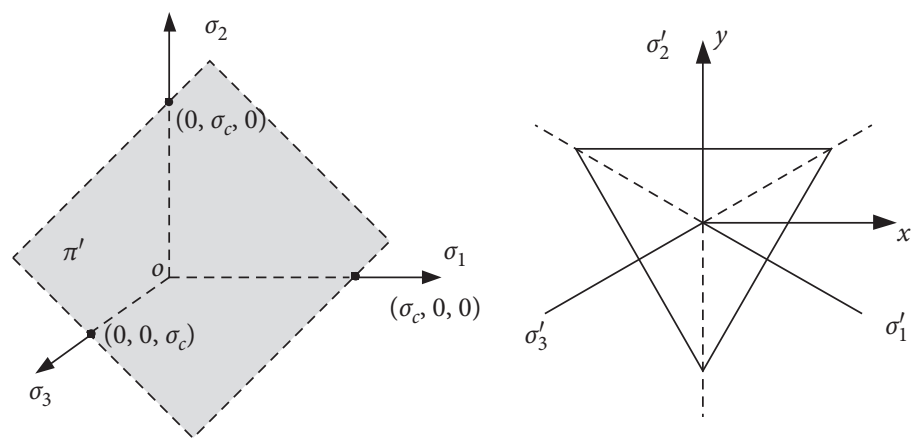

Figure 5: Yield curves on $\pi^{\prime}$-plane.

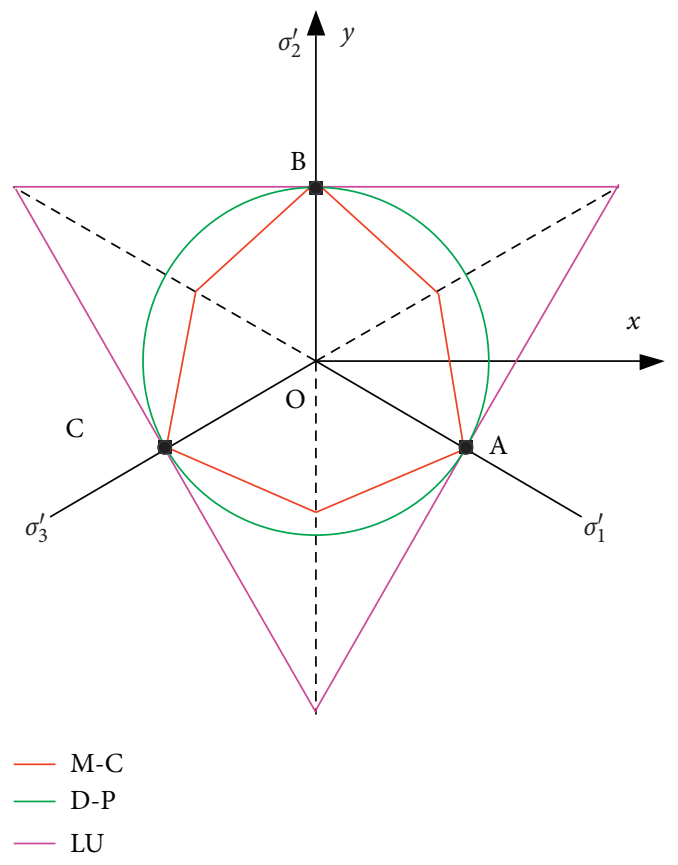

FIgURE 6: Yield curves of different criteria on $\pi^{\prime}$-plane.

As $\sigma_{2}=\sigma_{3}$, the criterion equation (21) can be rewritten as

$$
\sigma_{1}=\sigma_{c}+\frac{1-\mu_{c}}{\mu_{c}} \sigma_{3}
$$

Equation (31) is actually the maximum tensile strain criterion. The maximum tensile strain $\varepsilon_{3 \max }$ can be obtained from equation (6). For the uniaxial tensile test, $\varepsilon_{3 \max }=\varepsilon_{u}$. Combining equation (15), one has

$$
\varepsilon_{3 \max }=\frac{1}{E_{c}}\left[\sigma_{3}-\mu_{c}\left(\sigma_{1}+\sigma_{2}\right)\right]=\frac{\sigma_{t}}{E_{t}} .
$$

Substituting equation (18) into equation (32) gives the same result as equation (31). Thus, as $\sigma_{2}=\sigma_{3}$, equation (31) is the maximum tensile strain criterion. The data of conventional triaxial tests by Fujii can verify the criterion indirectly [15].

For most of the previous rock strength tests, one mainly focused on the yield strength $\sigma_{1}$ under different confining pressure $\sigma_{3}$. Poisson's ratio of uniaxial compression was seldom measured. Therefore, to verify the criterion of equation (31), the best-fit values of Poisson's ratio $\mu_{c}^{*}$ and UCS $\sigma_{c}^{*}$ were applied in the following process based on the experiment results $\left(\sigma_{1 i}, \sigma_{3 i}\right)(i=1,2, \ldots, n)$. The leastsquare methods were used herein to obtain the two fitting values:

$$
\begin{aligned}
\mu_{c}^{*} & =\frac{\sum_{i=1}^{n} \sigma_{3 i}^{2}-1 / n\left(\sum_{i=1}^{n} \sigma_{3 i}\right)^{2}}{\sum_{i=1}^{n} \sigma_{1 i} \sigma_{3 i}-1 / n \sum_{i=1}^{n} \sigma_{1 i} \sum_{i=1}^{n} \sigma_{3 i}+\sum_{i=1}^{n} \sigma_{3 i}^{2}-1 / n\left(\sum_{i=1}^{n} \sigma_{3 i}\right)^{2}} \\
\sigma_{c}^{*} & =1 / n \sum_{i=1}^{n} \sigma_{1 i}-\frac{1-\mu_{c}^{*}}{n \mu_{c}^{*}} \sum_{i=1}^{n} \sigma_{3 i} .
\end{aligned}
$$

In equation (31), substituting $\mu_{c}^{*}$ and $\sigma_{c}^{*}$ for $\mu_{c}$ and $\sigma_{c}$, respectively, we have

$$
\widehat{\sigma}_{1}=\sigma_{c}^{*}+\frac{1-\mu_{c}^{*}}{\mu_{c}^{*}} \sigma_{3},
$$

where $\widehat{\sigma}_{1}$ is the theoretical results, also known as the estimated value. The coefficient of determination $R^{2}$ describes the "goodness of fit" between the testing data and estimated values:

$$
R^{2}=1-\frac{\sum_{i=1}^{n}\left(\sigma_{1 i}-\widehat{\sigma}_{1 i}\right)^{2}}{\sum_{i=1}^{n}\left(\sigma_{1 i}-\bar{\sigma}_{1}\right)^{2}},
$$

where $\bar{\sigma}_{1}=(1 / n) \sum_{i=1}^{n} \sigma_{1 i}$ and $\widehat{\sigma}_{1 i}$ can be determined by equation (33).

The closer the value of $R^{2}$ is to 1 , the better the estimated $\widehat{\sigma}_{1}$ fits the testing data $\sigma_{1}$. Thus, if the value of $R^{2}$ by equation (36) is to 1 and the value of $\mu_{c}^{*}$ is within the range of rock material, we can say that the criterion is applicable to the conventional triaxial test.

Figures 7-10 illustrate the conventional triaxial test results of different rocks and the corresponding theoretical results of the criterion.

For the testing data of the 11 conventional triaxial tests, the values of $R^{2}$ are all close to 1 , with the minimum value of 0.9433 and the average value of 0.9745 . Moreover, calculations show that, for brittle rock, when the confining pressure is not very large, the mean relative error between the optimized $\sigma_{c}^{*}$ and the measured $\sigma_{c}$ is about $14.3 \%$, and 


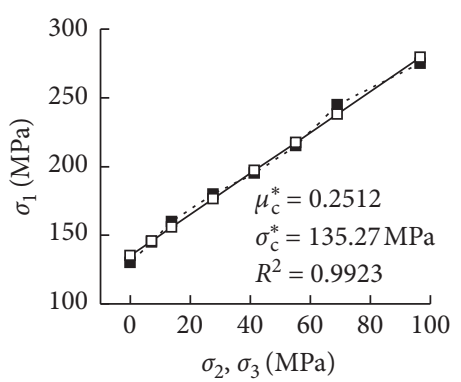

-. Experimental $\rightarrow-$ Theoretical

(a)

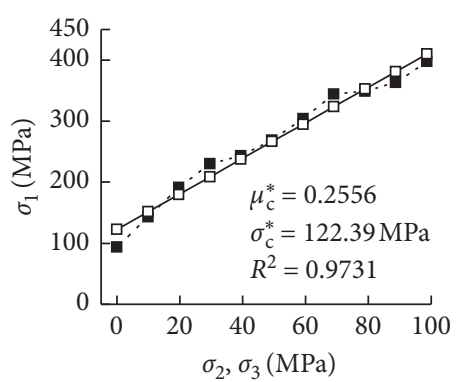

-. Experimental $\rightarrow \square$ Theoretical

(b)

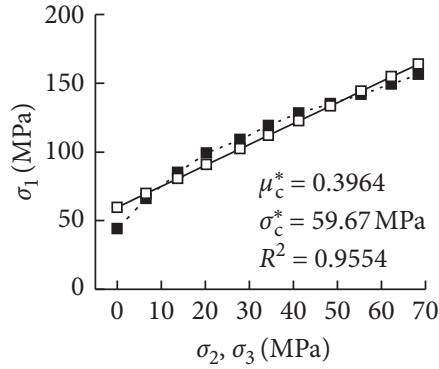

-. Experimental $\neg$ - Theoretical

(c)

Figure 7: Conventional triaxial test data and theoretical results of (a) Tennessee marble [29, 30], (b) Daye marble [6, 30], and (c) Indiana limestone [30, 31].

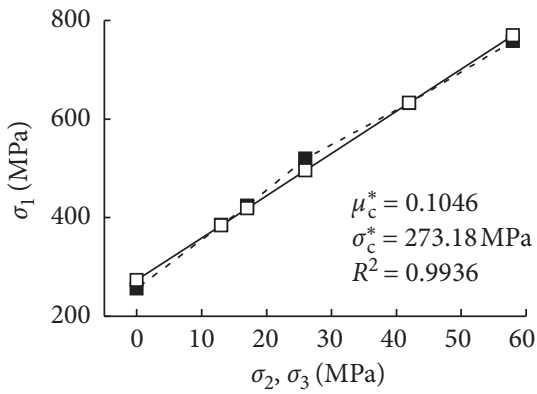

Experimental $\square-$ Theoretical

(a)

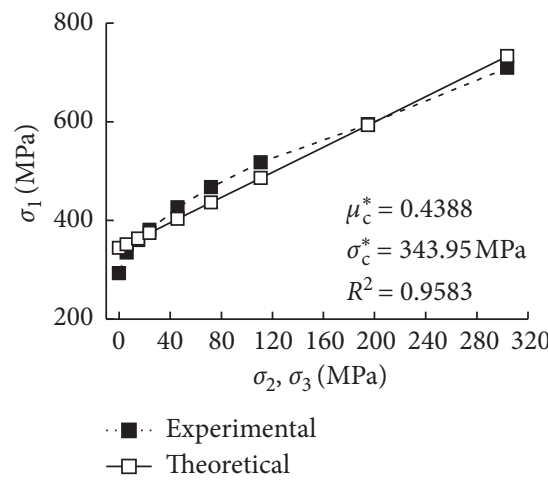

(c)

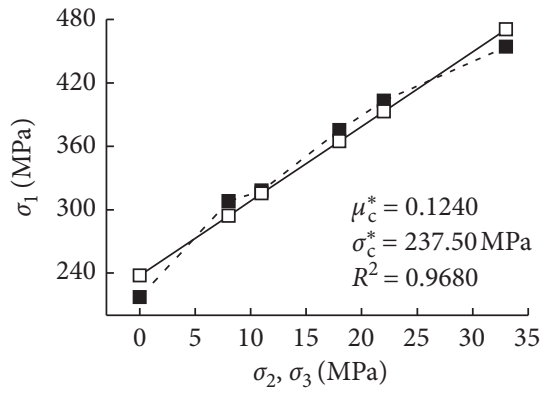

- Experimental $\rightarrow$ Theoretical

(b)

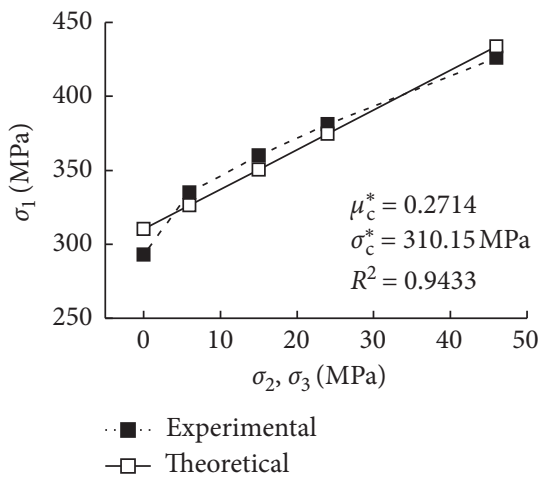

(d)

FIGURE 8: Conventional triaxial test data and theoretical results of (a) Westerly granite, (b) Dunham dolomite, (c) Solnhofen limestone, and (d) Solnhofen limestone [9].

most values of the optimized $\mu_{c}^{*}$ are between 0.16 and 0.3 , in the range of rock materials [35].

When the confining pressure is very large (see Figure $8(\mathrm{c})$ ), the theoretical results do not fit the testing data very well with the relative error around $18.2 \%$. Moreover, the optimized value of $\mu_{c}^{*}$ is 0.4388 , differing significantly from the value 0.2714 (see Figure $8(\mathrm{~d})$ ) and beyond the reasonable range of $0.16-0.3$. The reason may be that, under relatively high confining pressure, the application condition $\sigma_{2}+\sigma_{3}<2 \mu_{c}\left(\sigma_{1} /\left(1-\mu_{c}\right)\right)$ cannot be satisfied anymore, such as the last data in Figure 8(c) $\left(\sigma_{2}=\sigma_{3}=304 \mathrm{MPa}\right.$; $\left.\sigma_{1}=709 \mathrm{MPa}\right)$. Thus, the optimized value of $\mu_{c}^{*} 0.2714$ under lower confining pressure should be more reasonable.

Except the case of Figure 8(c), all the other 10 tests can be well explained by the criterion.

5.2. True Triaxial Tests. The triaxial test, in which all three principal stresses are different, is known as the true triaxial test. Similarly, as in Section 5.1, Poisson's ratio and UCS can 


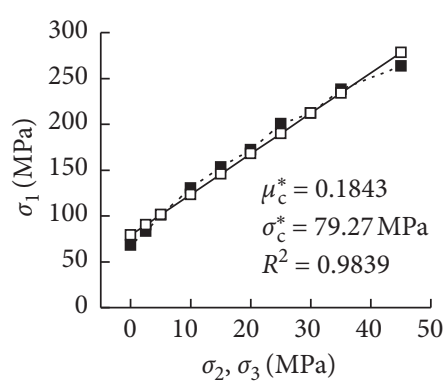

-. . Experimental $\rightarrow$ - Theoretical

(a)

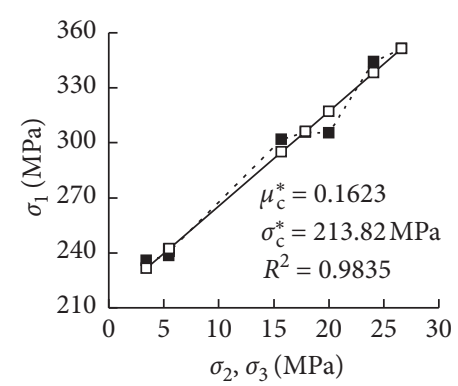

-.. Experimental $\rightarrow-$ Theoretical

(b)

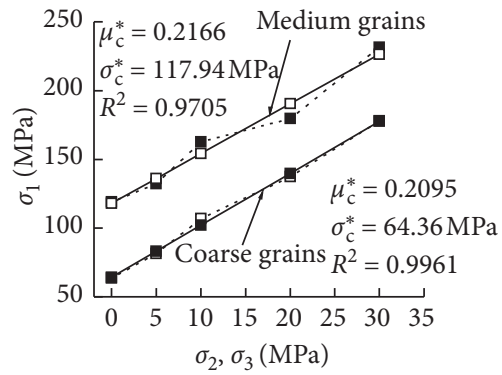

$\neg$ - Experimental

-. Theoretical

Figure 9: Conventional triaxial test data and theoretical results of (a) red sandstone [32], (b) Jinping marble [33], and (c) marble with medium and coarse grains [34].

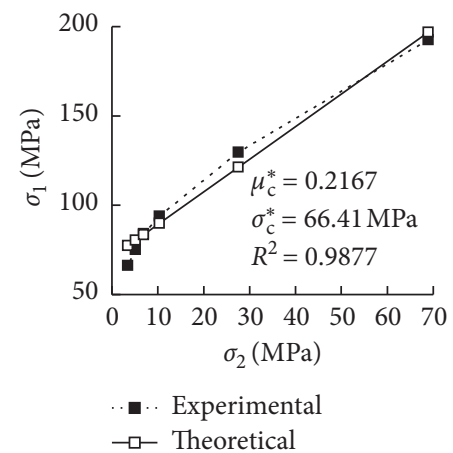

(a)

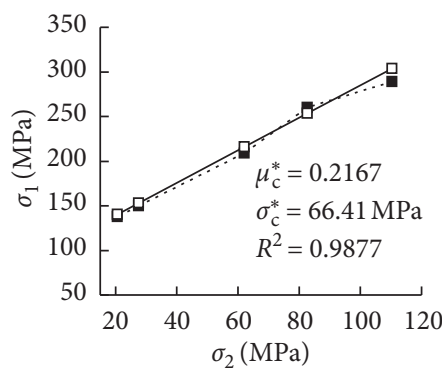

-.. Experimental $\rightarrow-$ Theoretical

(d)

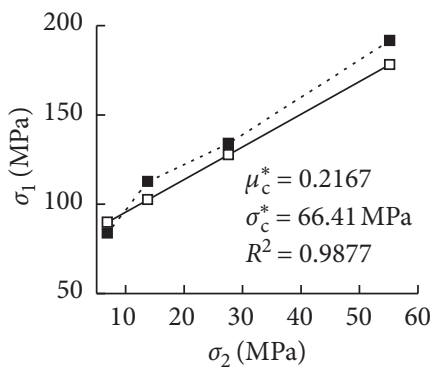

a.. Experimental $\rightarrow-$ Theoretical

(b)

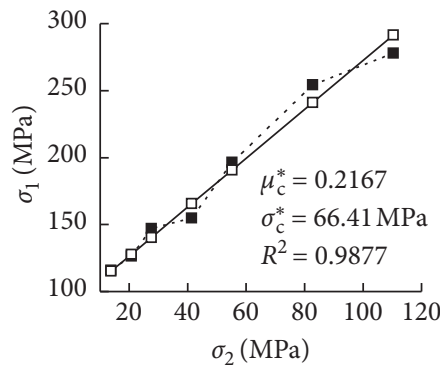

Experimental $\rightarrow$ Theoretical

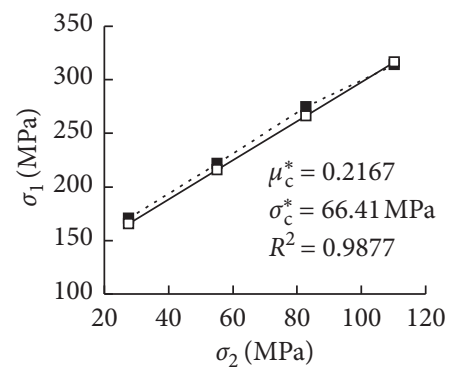

-.. Experimental $\rightarrow-$ Theoretical

(e)

Figure 10: True triaxial tests data and theoretical results of coarse-grained dense marble [36]. (a) $\sigma_{3}=3.45 \mathrm{MPa}$. (b) $\sigma_{3}=6.89 \mathrm{MPa}$. (c) $\sigma_{3}=13.79 \mathrm{MPa}$. (d) $\sigma_{3}=20.68 \mathrm{MPa}$. (e) $\sigma_{3}=27.58 \mathrm{MPa}$.

be determined using the testing data $\left(\sigma_{1 i}, \sigma_{2 i}, \sigma_{3 i}\right)(i=1$, $2, \cdots, n)$. The optimized values of $\mu_{c}^{*}$ and $\sigma_{c}^{*}$ in equation (21) can be obtained by substituting $\sigma_{3 i}$ into $\left(\sigma_{2 i}+\sigma_{3 i}\right) / 2$ in equations (35) and (36). Substituting $\mu_{c}$ and $\sigma_{c}$ into $\mu_{c}^{*}$ and $\sigma_{c}^{*}$, respectively, we have

$$
\widehat{\sigma}_{1}=\sigma_{c}^{*}+\frac{1-\mu_{c}^{*}}{2 \mu_{c}^{*}}\left(\sigma_{2}+\sigma_{3}\right) .
$$

The coefficient of determination $R^{2}$ is still calculated using equation (36), in which $\widehat{\sigma}_{1 i}$ should be calculated using equation (37). The values of $R^{2}$ and $\mu_{c}^{*}$ are used to measure whether the proposed criterion is applicable to the true triaxial tests.

Example 1. Coarse-grained dense marble.

In Table 1, 30 datasets of true triaxial tests are listed, 4 of which are special with $\sigma_{1}$ and $\sigma_{2}$ in negative correlation (highlighted in bold). As discussed above, for the criterion, only those positive related data of $\left\{\sigma_{1}, \sigma_{2}\right\}$ can be used as calculating the optimized values of $\mu_{c}^{*}$ and $\sigma_{c}^{*}$. Moreover, the rest 26 datasets can satisfy the application requirement (i.e., $\left.\sigma_{2}+\sigma_{3}<2\left(\left(\mu_{c}^{*} \sigma_{1}\right) / 1-\mu_{c}^{*}\right)\right)$. Thus, based on the 26 valid 


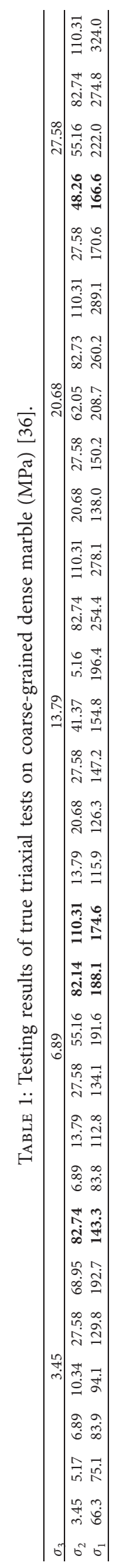




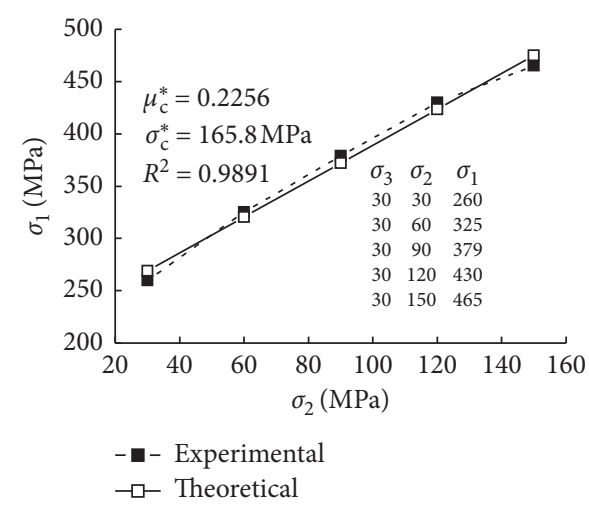

(a)

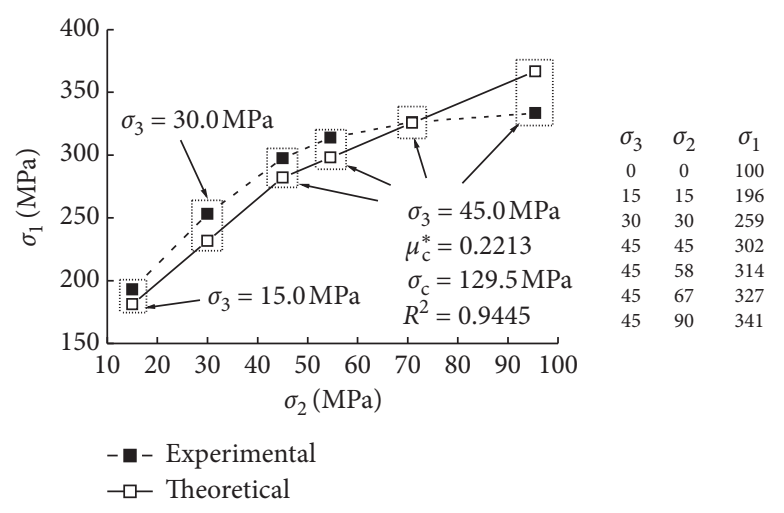

(b)

Figure 11: True triaxial test data and theoretical results of (a) Laxiwa marble [37] and (b) trachyte [9].

datasets, we have $\mu_{c}^{*}=0.2167, \sigma_{c}^{*}=66.41 \mathrm{MPa}$, and $R^{2}=$ 0.9877 . The experimental and theoretical results of coarsegrained dense marble are illustrated in Figure 10.

Example 2. Laxiwa marble [37] and trachyte [9].

The experimental and theoretical results of Laxiwa marble [37] and trachyte [9] are illustrated in Figure 11.

For Laxiwa marble, all five testing data are valid, with $\sigma_{2}$ and $\sigma_{1}$ in positive correlation, and $\mu_{c}^{*}=0.2256$, $\sigma_{c}^{*}=165.8 \mathrm{MPa}$, and $R^{2}=0.9891$. For trachyte, only the seven datasets as $\sigma_{3} \leq 45 \mathrm{MPa}$ are valid, and the corresponding results are $\mu_{c}^{*}=0.2213, \sigma_{c}^{*}=129.5 \mathrm{MPa}$, and $R^{2}=0.9445$.

The comparisons of the two examples indicate that the testing and theoretical results are in good agreement (see Figures 9 and 11) with $R^{2}=0.9877$ and 0.9445 , respectively.

\section{Conclusions}

A new extension failure criterion was proposed based on the extension rate of the plane of the maximum principal stress. It is different from the maximum strain criterion which cannot reflect the main properties of rock failure. Although the maximum strain criterion is seldom using for rock material, it can well explain the phenomenon in the conventional triaxial test that the critical lateral tensile strain for brittle rock almost keeps as a constant at failure [15]. For the special conventional triaxial tests, the proposed criterion is exactly the same as the maximum strain criterion.

The proposed criterion, $\sigma_{1}=\sigma_{c}+\left(1-\mu_{c} / 2 \mu_{c}\right)\left(\sigma_{2}+\sigma_{3}\right)$, can reflect the phenomenon that the stress $\sigma_{1}$ at failure increases with the other two principal stresses $\sigma_{2}$ and $\sigma_{3}$. But the criterion is only suitable as $\sigma_{2}+\sigma_{3}<2\left(\mu_{c} \sigma_{1} /\left(1-\mu_{c}\right)\right)$. As the lateral pressure is relatively great (i.e., $\sigma_{2}+\sigma_{3}>$ $\left.2\left(\mu_{c} \sigma_{1} / 1-\mu_{c}\right)\right)$, the specimen will be in the state of volume shrinkage and the failure is not tensile anymore, so the criterion is not applicable anymore either.

The criterion expressed by the principal stresses has concise expressions and clear physical background. The fitting results based on this criterion agree with the test data well. It was also compared with the Mohr-Coulomb and Drucker-Prager criteria.
The paper only discussed the triaxial compressive condition. For this case, two parameters are contained in the new criterion, that is, the compressive Poisson's ratio and uniaxial compression strength. In geotechnical engineering, the principal stresses may be tensile, and the criterion of that can also be given based on a similar way.

$\begin{array}{ll}\text { Abbreviations } \\ \sigma_{1}: & \text { Maximum principal compressive stress } \\ \sigma_{2}: & \text { Intermediate principal compressive stress } \\ \sigma_{3}: & \text { Minimum principal compressive stress } \\ \sigma: & \text { Normal stress on a certain plane } \\ \tau: & \text { Shear stress on a certain plane } \\ \sigma^{c}: & \text { Uniaxial compression stress } \\ \sigma^{t}: & \text { Uniaxial tensile stress } \\ \sigma_{c}: & \text { Uniaxial compressive strength (UCS) } \\ \sigma_{t}: & \text { Uniaxial tensile strength (UTS) } \\ \varepsilon_{1}: & \text { Maximum principal tensile strain } \\ \varepsilon_{2}: & \text { Intermediate principal tensile strain } \\ \varepsilon_{3}: & \text { Minimum principal strain } \\ \varepsilon_{u}: & \text { Critical tensile strain corresponding to } \sigma_{t} \\ \sigma_{c}^{0}: & \text { Hydrostatic compressive stress on } \\ \sigma_{t}^{0}: & \text { yielding } \\ E_{c}: & \text { Hydrostatic tensile stress on yielding } \\ E_{t}: & \text { Young's modulus for compression } \\ \mu_{c}: & \text { Young's modulus for tension } \\ \mu_{t}: & \text { Poisson's ratio for compression } \\ c: & \text { Poisson's ratio for tension } \\ \varphi: & \text { Cohesion of Mohr-Coulomb material } \\ l: & \text { Friction angle of Mohr-Coulomb material } \\ \Delta l_{1}, \Delta l_{2}: & \text { Side length of the } \sigma_{1} \text {-plane } \\ S: & \text { Increase of side length } \\ \Delta S: & \text { Area of the } \sigma_{1} \text {-plane } \\ S / S=\varepsilon_{1}+\varepsilon_{2}: & \text { Increase of the area } \\ \pi, \pi^{\prime}: & \text { Extension rate of the } \sigma_{1} \text {-plane } \\ x, y: & \text { Deviatoric planes } \\ \sigma_{1}^{\prime}, \sigma_{2}^{\prime}, \sigma_{3}^{\prime}: & \text { Cartesian coordinates of yield curves in } \\ & \pi^{\prime} \text {-plane } \\ \text { Projections of } \sigma_{1}, \sigma_{2}, \sigma_{3} \text { in } \pi^{\prime} \text {-plane } \\ \end{array}$




$\begin{array}{ll}\sigma_{1 i}, \sigma_{2 i}, \sigma_{3 i}(i=1,2, \ldots, n): \begin{array}{l}\text { Experiment data } \\ \mu_{c}^{*}:\end{array} & \text { Best-fit values of Poisson's } \\ & \text { ratio } \\ \sigma_{c}^{*}: & \text { Best-fit values of uniaxial } \\ & \text { compressive strength } \\ \widehat{\sigma}_{1}: & \text { Estimated value of } \sigma_{1} \\ R^{2}: & \text { Coefficient of determination. }\end{array}$

\section{Data Availability}

Previously reported data were used to support this study. These prior studies (and datasets) are cited at relevant places within the text as references [1-37].

\section{Conflicts of Interest}

The authors declare that they have no conflicts of interest.

\section{Acknowledgments}

This study was funded by the National Natural Science Foundation of China (Grants nos. 51974124 and 51704117).

\section{References}

[1] C. A. Coulomb, "Application des règles de maxima et minima à quelques problèkme s de statiquerelatifs à 1'Architecture,” Academie Royale des Sciences, vol. 7, pp. 343-382, 1773.

[2] O. Mohr, "Welche Umstände bedingen die Elastizitätsgrenze und den Bruch eines Materials?" Zeitschrift des Vereins Deutscher Ingenieure, vol. 46, pp. 1524-1530, 1900.

[3] J. C. Jaeger, N. G. Cook, and R. Zimmerman, Fundamentals of Rock Mechanics, John Wiley \& Sons, Hoboken, NJ, USA, 2009.

[4] E. Hoek and E. T. Brown, "Empirical strength criterion for rock masses," Journal of Geotechnical and Geoenvironmental Engineering, vol. 106, pp. 1013-1035, 1980.

[5] X. Si, F. Gong, X. Li, S. Wang, and S. Luo, "Dynamic MohrCoulomb and Hoek-Brown strength criteria of sandstone at high strain rates," International Journal of Rock Mechanics and Mining Sciences, vol. 115, pp. 48-59, 2019.

[6] M. Singh and K. S. Rao, "Bearing capacity of shallow foundations in anisotropic non-hoek-Brown rock masses," Journal of Geotechnical and Geoenvironmental Engineering, vol. 131, no. 8, pp. 1014-1023, 2005.

[7] F. Gong, X. Si, X. Li, and M. Tao, "Rock dynamic Mohr-Coulomb and Hoek-brown criteria based on strain rate effect," The Chinese Journal of Nonferrous Metals, vol. 26, no. 8, pp. 1763-1773, 2016.

[8] L. Ma, Z. Li, M. Wang, J. Wu, and G. Li, "Applicability of a new modified explicit three-dimensional Hoek-Brown failure criterion to eight rocks," International Journal of Rock Mechanics and Mining Sciences, vol. 133, Article ID 104311, 2020.

[9] K. Mogi, Experimental Rock Mechanics, Taylor \& Francis, London, UK, 2007.

[10] D. C. Drucker and W. Prager, "Soil mechanics and plastic analysis or limit design," Quarterly of Applied Mathematics, vol. 10, no. 2, pp. 157-165, 1952.

[11] G. A. Wiebols and N. G. W. Cook, "An energy criterion for the strength of rock in polyaxial compression," International Journal of Rock Mechanics and Mining Sciences \& Geomechanics Abstracts, vol. 5, no. 6, pp. 529-549, 1968.
[12] A. M. Al-Ajmi and R. W. Zimmerman, "Relation between the Mogi and the coulomb failure criteria," International Journal of Rock Mechanics and Mining Sciences, vol. 42, no. 3, pp. 431-439, 2005.

[13] M. You, "True-triaxial strength criteria for rock," International Journal of Rock Mechanics and Mining Sciences, vol. 46, no. 1, pp. 115-127, 2009.

[14] D. K. Hallbauer, H. Wagner, and N. G. W. Cook, "Some observations concerning the microscopic and mechanical behaviour of quartzite specimens in stiff, triaxial compression tests," International Journal of Rock Mechanics and Mining Sciences \& Geomechanics Abstracts, vol. 10, no. 6, pp. 713-726, 1973.

[15] Y. Fujii, T. Kiyama, Y. Ishijima, and J. Kodama, "Examination of a rock failure criterion based on circumferential tensile strain," Pure and Applied Geophysics, vol. 152, no. 3, pp. 551-577, 1998.

[16] E. Hoek and C. D. Martin, "Fracture initiation and propagation in intact rock-a review," Journal of Rock Mechanics and Geotechnical Engineering, vol. 6, no. 4, pp. 287-300, 2014.

[17] I. Hawkes, M. Mellor, and S. Gariepy, "Deformation of rocks under uniaxial tension," International Journal of Rock Mechanics and Mining Sciences \& Geomechanics Abstracts, vol. 10, no. 6, pp. 493-507, 1973.

[18] B. C. Haimson and T. M. Tharp, "Stresses around boreholes in bilinear elastic rock," Society of Petroleum Engineers Journal, vol. 14, no. 2, pp. 145-151, 1974.

[19] B. Stimpson and R. Chen, "Measurement of rock elastic moduli in tension and in compression and its practical significance," Canadian Geotechnical Journal, vol. 30, no. 2, pp. 338-347, 1993.

[20] X. B. Yu, Q. R. Wang, X. Y. Li, Q. Xie, Y. K. Na, and Z. P. Song, "Experimental research on deformation of rocks in direct tension and compression," Rock and Soil Mechanics, vol. 29, no. 1, pp. 18-22, 2008.

[21] D. Y. Li, X. B. Li, and C. Li, "Experimental studies of mechanical properties of two rocks under direct compression and tension," Chinese Journal of Rock Mechanics and Engineering, vol. 29, no. 3, pp. 624-632, 2010.

[22] K. Fuenkajorn and S. Klanphumeesri, "Laboratory determination of direct tensile strength and deformability of intact rocks," Geotechnical Testing Journal, vol. 34, pp. 97-102, 2010.

[23] W. Jiang, "Comparative study on rocks in different tensile and compressive tests," thesis of Master Degree, Nanjing University, Nanjing, China, 2014.

[24] S. B. Yuan, "Experimental and numerical analysis study on direct tensile test of hard brittle rocks," thesis of Master Degree, Shandong University, Jinan, China, 2016.

[25] S. Ambartsumyan, Elasticity Theory of Different Modulus, China Railway Press, Beijing, China, 1986.

[26] A. Coviello, R. Lagioia, and R. Nova, "On the measurement of the tensile strength of soft rocks," Rock Mechanics and Rock Engineering, vol. 38, no. 4, pp. 251-273, 2005.

[27] J. Hudson, E. Brown, and F. Rummel, "The controlled failure of rock discs and rings loaded in diametral compression," International Journal of Rock Mechanics and Mining Sciences \& Geomechanics Abstracts, vol. 9, no. 12, pp. 2241-2248, 1972.

[28] P. R. Sheorey, A. K. Biswas, and V. D. Choubey, "An empirical failure criterion for rocks and jointed rock masses," Engineering Geology, vol. 26, no. 2, pp. 141-159, 1989.

[29] W. R. Wawersik and C. Fairhurst, "A study of brittle rock fracture in laboratory compression experiments," International Journal of Rock Mechanics and Mining Sciences \& Geomechanics Abstracts, vol. 7, no. 5, pp. 561-575, 1970. 
[30] J. Peng, G. Rong, M. Cai, X. Wang, and C. Zhou, "An empirical failure criterion for intact rocks," Rock Mechanics and Rock Engineering, vol. 47, no. 2, pp. 347-356, 2014.

[31] A. E. Schwartz, "Failure of rock in the triaxial shear test," in Proceedings of the Sixth Symposium on Rock Mechanics, pp. 109-151, Rolla, MO, USA, October 1964.

[32] C. D. Su and Y. S. Fu, "Experimental study of triaxial compression deformation and strength characteristics of red sandstone," Chinese Journal of Rock Mechanics \& Engineering, vol. 33, no. 1, pp. 3164-3169, 2014.

[33] X. P. Li, H. Zhao, and T. L. Xiao, "Research on constitutive model of Jinping marble under unloading conditions and numerical simulation," Rock and SoilMechanics, vol. 33, no. 2, pp. 401-407, 2012.

[34] S. Yang, C. Su, and W. Xu, "Experimental investigation on strength and deformation properties of marble under conventional triaxial compression," Rock and Soil Mechanics, vol. 26, no. 3, pp. 475-478, 2005.

[35] H. Gercek, "Poisson's ratio values for rocks," International Journal of Rock Mechanics and Mining Sciences, vol. 44, no. 1, pp. 1-13, 2007.

[36] P. Michelis, "Polyaxial yielding of granular rock," Journal of Engineering Mechanics, vol. 111, no. 8, pp. 1049-1066, 1985.

[37] M. H. Yu, Twin-shear Theory and its Application, Science Press, Beijing, China, 1998. 